\title{
The postnatal development of lymphoreticular aggregates and lymph nodes in infants' lungs
}

\author{
JOHN L. EMERY AND FAY DINSDALE \\ From the Department of Developmental Pathology and Children's Hospital, Sheffield
}

SYNOPSIS A quantitative study has been made of standardized sections taken from the right middle lobe of 316 children's lungs. Lungs showing no pathological state showed that lymph nodes are present at birth and these increase in prominence if not in numbers throughout the first year after birth. Lymphoreticular aggregates are not present among the alveoli of the normal infant lung at birth. These first appear around a week after birth and progressively increase in number throughout infancy and early childhood, being almost universally present by the age of 5 years. The development of peripheral lymphoreticular aggregates in the lungs of infants appears to be entirely environmentally and antigenically stimulated.

Routine microscopy reveals great variability in the amount and distribution of lymphoid tissue in the lungs of children. Quantitative studies of lymphoreticular tissue in lungs are not available and Yoffey and Courtice (1970) make the statement 'lungs may contain quite appreciable amounts of lymphoid tissue which we are unable to quantitate'.

The general distribution of the lymph drainage of the lung has been well described (Miller, 1911; Harvey and Zimmerman, 1935; Tobin, 1957; van Hayek, 1960; Collet and Policard, 1962; Bastianini, 1967) and recently the valvular component has been beautifully illustrated by Lauweryns (1971). The presence of extensive lymphoreticular aggregates in the lung quite distinct from lymph nodes is well known and has been studied in pigs by Jelinek and Jelinkova (1957).

Jericho, Derbyshire, and Jones (1971) and Jericho, Austwick, Hodges, and Dixon (1971), using newborn pigs, showed that the postnatal development of lymphoreticular aggregates within the lung tissue depends on inhaled antigenic stimulants in the form of bacteria, viruses, or parasites, and that such aggregates do not develop in response to inorganic material such as carbon particles. The question thus arises as to whether or not the same applies to the newborn infant and child.

For this reason a study has been carried out on the lungs of children coming to necropsy to estimate the variability in the amount and distribution of lymphoreticular aggregates. We have extended a quantitative

Received for publication 3 May 1973. method previously used in assessing the development of the lung (Emery and Wilcox, 1966) and we have applied histological criteria similar to those used by Jericho in pigs (Jericho, 1970).

\section{Material and Methods}

\section{MATERIAL}

It has been our routine practice to retain the whole of the right middle lobe of the lungs of all children coming to necropsy. After fixation a standard block is taken, completely traversing the lobe at a distance half way from the root of the lobe to the inferior corners and to the point at which the superior border leaves the general pleural surface. The points of measurement and site of taking the block are illustrated in figures 1 and 2. This block is sectioned and provides a standard sample of a lung of any size and age. The whole of such sections stained with haematoxylin and eosin and Masson's trichrome were scanned using an automatic stage microscope and the total lymphatic and lymphoreticular elements recorded.

A sequential series of 316 lungs was surveyed, the observer having no knowledge of the age, state or cause of death of the child.

The lymphatic tissues were classified according to their structure, location, and size, as illustrated in figs $3,4,5$, and 6 , and such figures were held for constant reference while scoring was being carried out.

The reproducibility of counts appeared to depend more on the quality of the section and its freedom 


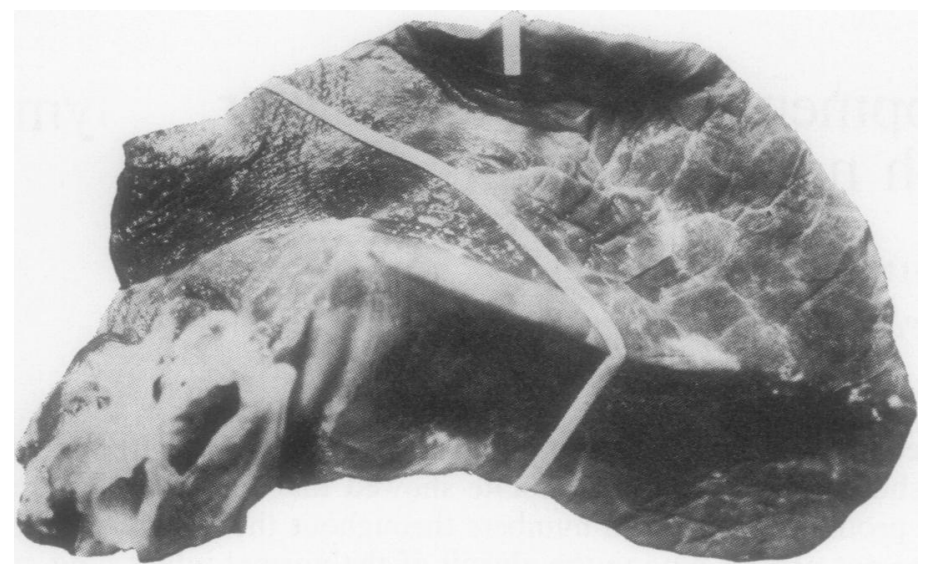

Fig 1 A right middle lobe showing a band of white paper covering the block taken for survey. The white strip at the top indicates the pleural angle used for measuring the mid point of the upper border of the lobe $(\times 1 \cdot 5)$.

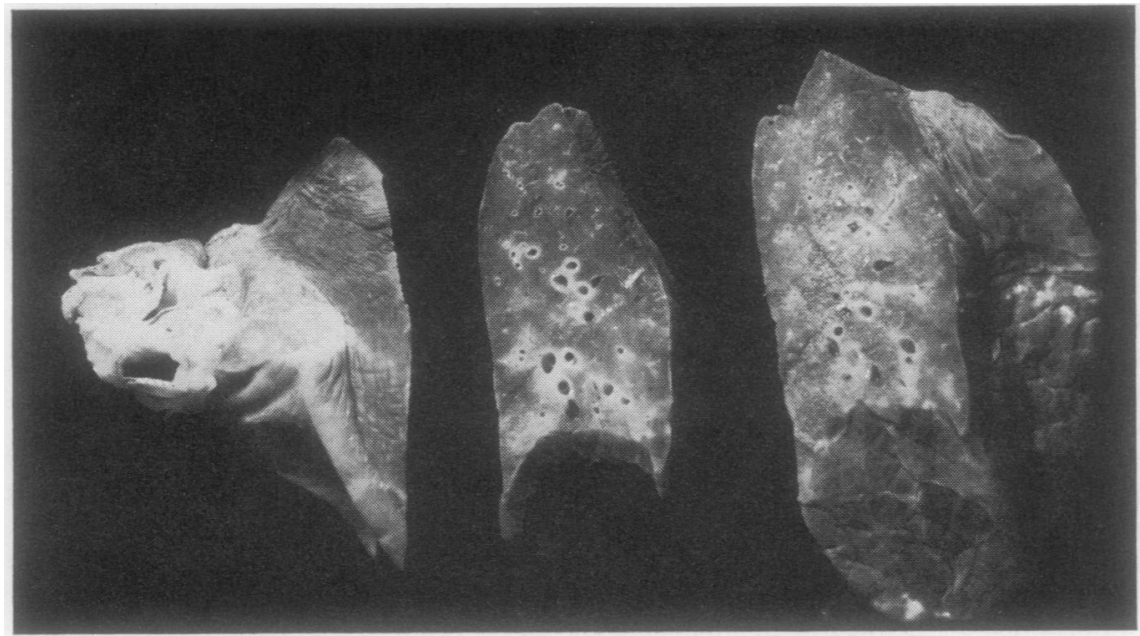

Fig 2 A lobe after blocking showing the standard block $\times 1)$.

from pathological processes than on errors of structure identification.

The lymphatic structures in the lung fall into two groups.

\section{Lymph nodes}

Lymph nodes, having a definite nodal structure with a peripheral sinus and a central blood vessel and recognized more on their structure than on their cell populations (Bryant and Shifrine, 1972), show great variation in the number of lymphocytes, and it is possible to identify them when they contain only a very few lymphocytes. These nodes occur in a variety of sites-bronchial, peribronchial, connective tissue and pleural-but never among alveoli.

\section{Lymphoreticular aggregates}

These consist of collections of cells chiefly lympho- cytes but with occasional eosinophils and plasma cells. They frequently have traversing capillaries but on reticulin staining show no nodal or sinusoidal structure (fig 7). They may abut and be partly surrounded by a lymph sinus but the sinus does not $\odot$ form part of the structure and there does not appear $N$ to be a lymph circulation system through them.

These also occur at different sites, among alveoli, 0 in the connective tissue adjacent to alveoli, in the pleura, between terminal bronchioles and their $\frac{C}{\mathscr{D}}$ accompanying arteries, and associated with the $\stackrel{\leftrightarrow}{?}$ epithelium of bronchi.

Independently of this scoring, all of the sections were re-examined together with the sections from $\stackrel{\odot}{\varnothing}$ the other lobes of the lungs and the complete $\overrightarrow{\mathbb{D}}$ necropsy report, and on this basis 103 lungs con- taining evidence of lung pathology-pneumonia, bronchitis, or old thromboses-were excluded as 


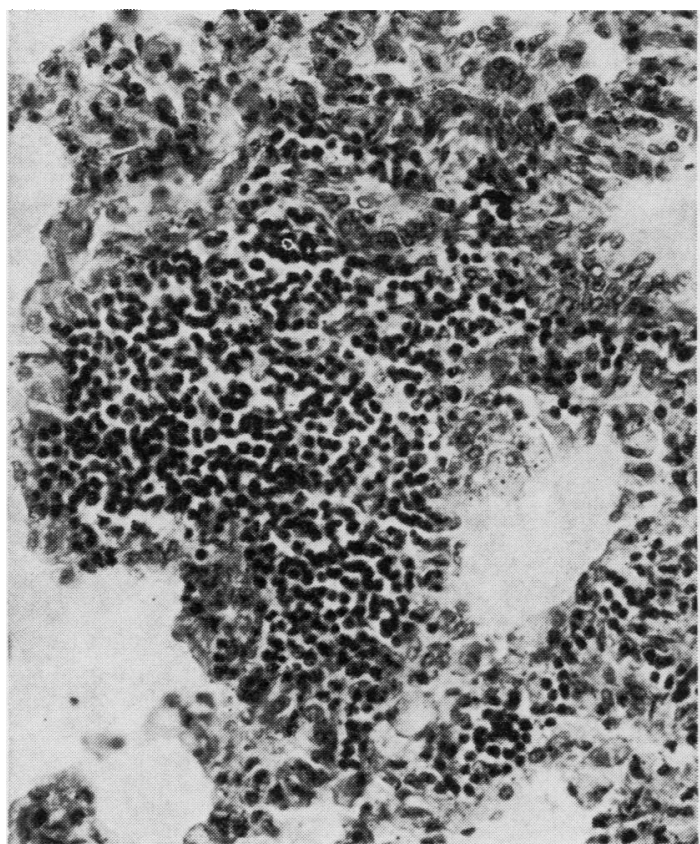

Fig 3

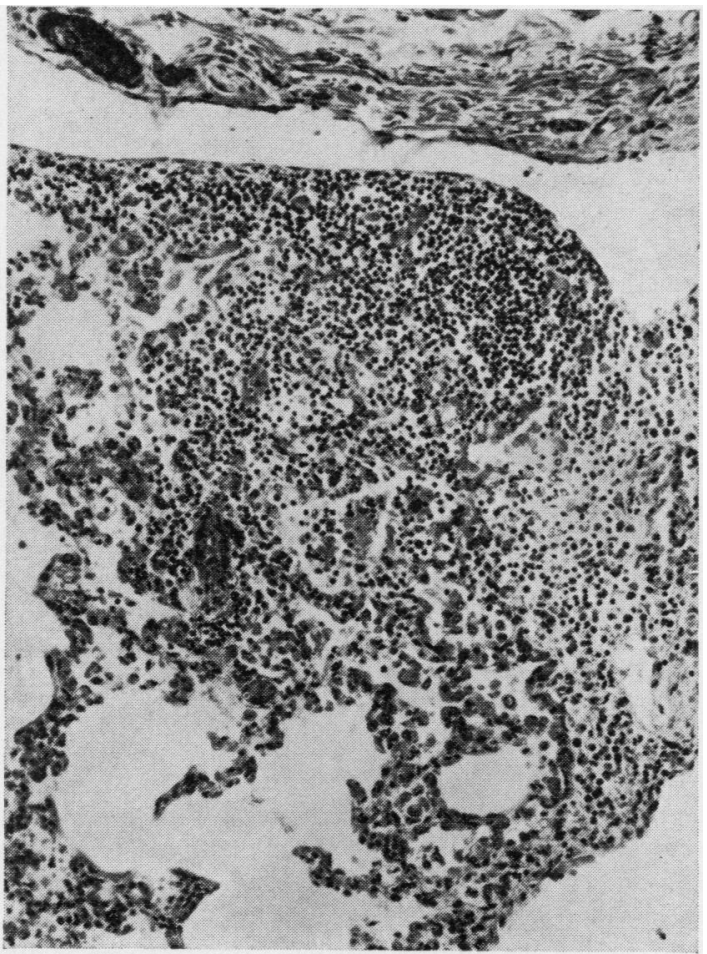

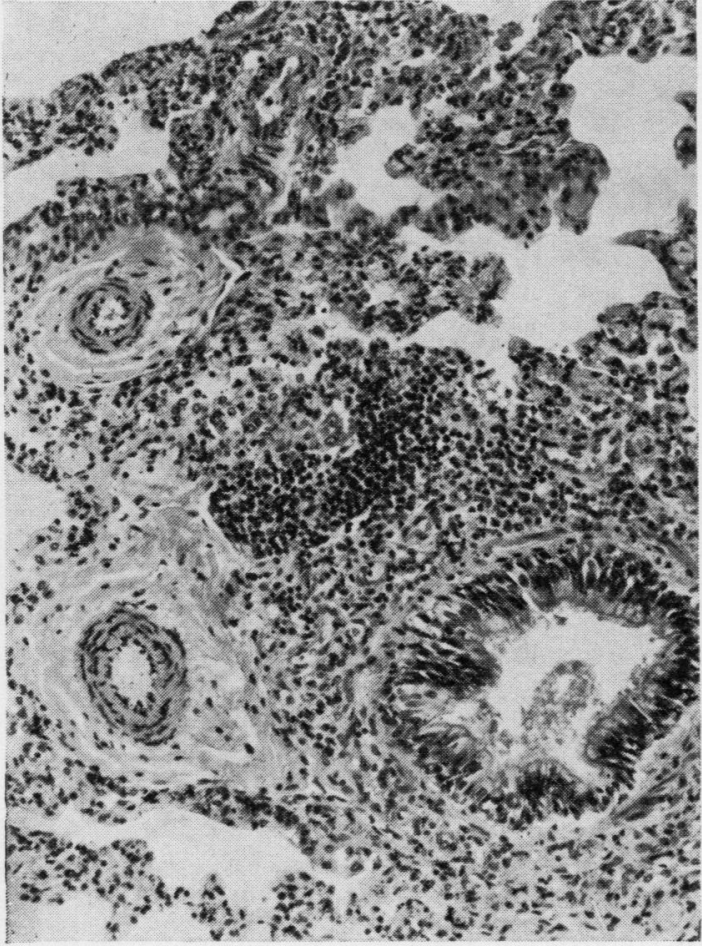

Fig 4

Fig 3 A lymphoreticular aggregate situated among alveoli. Note how some of the local alveolar walls have plumped up lining cells $(H \& E \times 315)$.

Fig 4 A lymphoreticular aggregate lying adjacent to a terminal bronchiole and its accompanying arteries $(H \& E \times 200)$.

Fig 5 An aggregate situated at the edge of a lung lobule - note its situation beside a lymph channel and the presence of apparent free cells in local alveoli $(H \& E$ $\times 200$ ).

Fig 5 


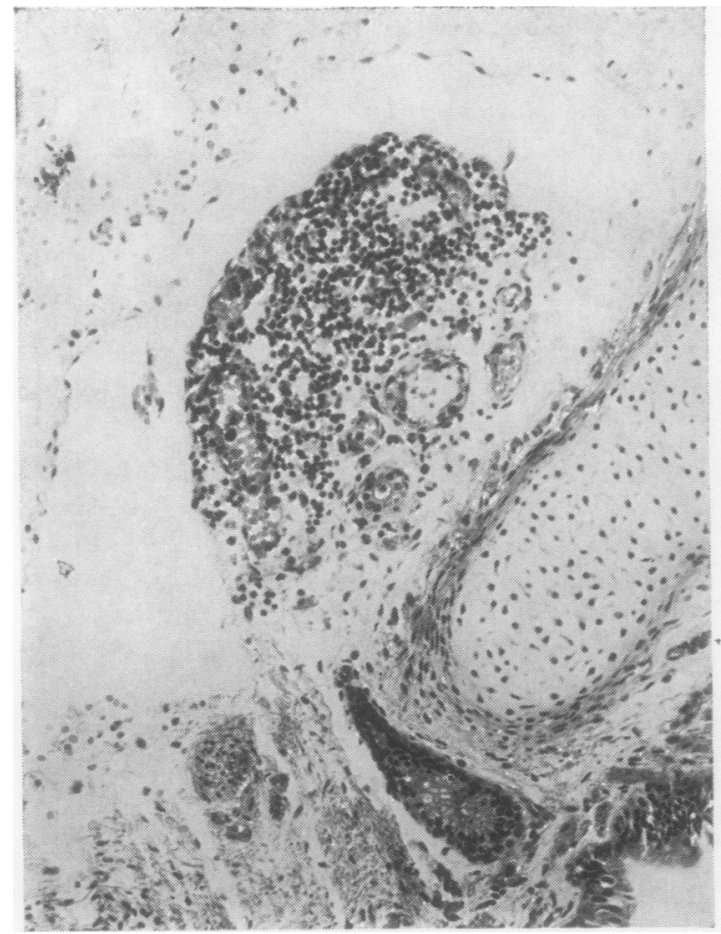

Fig 6

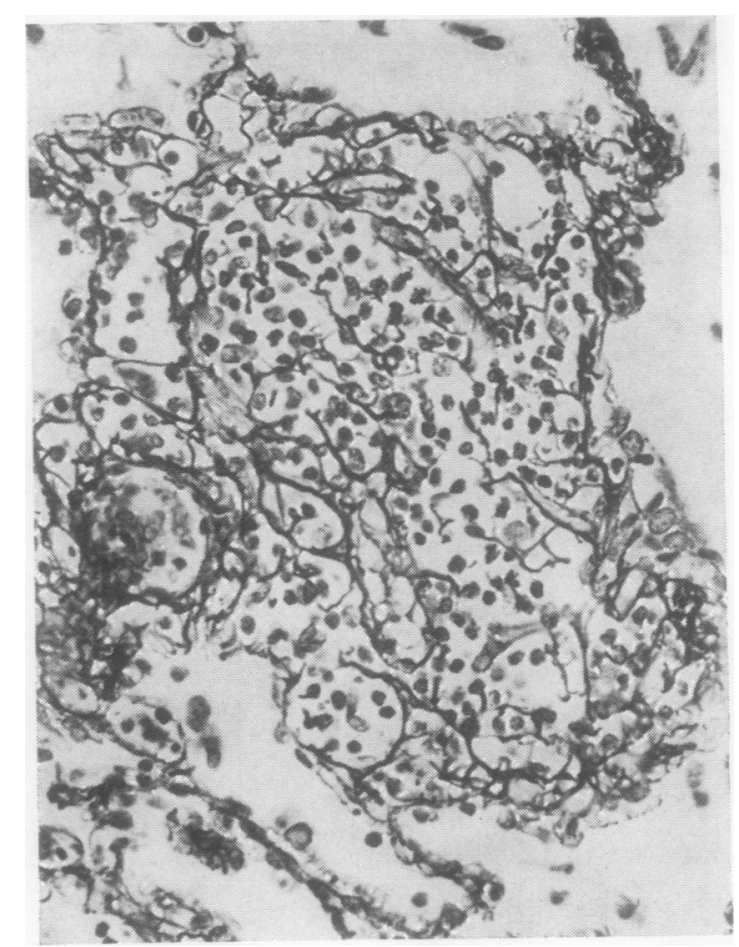

Fig 7

Fig 6 A lymphoreticular structure situated deep to the cartilage of a bronchus and surrounded by a lymph channel. The local bronchial gland almost certainly has an extension terminating in this structure. Such structures occur in young infants at sites that seem later to bear lymph nodes and these have been classified as nodes $(\times 135)$.

Fig 7 A lymphoreticular aggregate among alveoli stained to show the reticulin structure $(\times 375)$.

were those in which there was definite evidence in the trachea or larynx that death was due to a respiratory tract infection. The presence of a small amount of oedema was not used as an indication for eliminating the lung nor were small areas of collapsed alveoli or the occasional free epithelial cell within the alveoli. The presence or absence of lymphoreticular aggregates in isolation was not used as an assessment criterion. No distinction was made between children found unexpectedly dead or otherwise. With the children dying within the neonatal period the sections of the costochondral junction and thymus were re-surveyed with the whole postmortem protocol to exclude the presence of intrauterine infection.

There remained a total of 213 lungs which showed no demonstrable lung pathology and these were considered to be presumptively normal. Of these, 119 were children who had been found unexpectedly dead, and 94 died from other causes.

\section{Results}

The general results are shown in fig 8 and in the table. There is a progressive and steep postnatal rise in the number of both lymph nodes and in lymphoreticular aggregates. This increase is about tenfold during the first year. The number of reticular aggregates is about seven times that of the number of lymph nodes. The ratio of the two groups of structures appears to remain the same throughout this period The average number of nodes seen in the standard sections varied from 0.15 at birth to 2.9 at 3 years.

The lymph nodes in infant lungs, particularly in children below the age of 3 months, only rarely show the presence of Flemming centres. Within the whole series structural nodes with Flemming centres were seen in 11 only. The youngest was a child with pneumonia dying at $2 \frac{1}{2}$ months and the youngest child in the apparently normal lungs was aged 10 months. 


\begin{tabular}{|c|c|c|c|c|c|c|c|c|c|c|c|}
\hline \multirow[t]{3}{*}{ No. } & \multirow{2}{*}{\multicolumn{2}{|c|}{$\begin{array}{l}\text { Cases Studied } \\
\text { Age }\end{array}$}} & \multirow{2}{*}{\multicolumn{2}{|c|}{$\frac{\text { Lymph Nodes }}{\text { Total }}$}} & \multicolumn{7}{|c|}{ Lymphoreticular Aggregates } \\
\hline & & & & & \multicolumn{2}{|l|}{ Total } & \multicolumn{5}{|c|}{ Percentage of Lungs Showing Aggregates in Different Areas } \\
\hline & Mean & (Range) & $\begin{array}{l}\text { Mean/ } \\
\text { Section }\end{array}$ & (Range) & $\begin{array}{l}\text { Meanl } \\
\text { Section }\end{array}$ & (Range) & Alveolar & $\begin{array}{l}\text { Connective } \\
\text { Tissue }\end{array}$ & Pleural & $\begin{array}{l}\text { Bronchiolar } \\
\text { Blood Vessel }\end{array}$ & Bronchial \\
\hline $\begin{array}{r}21 \\
47 \\
23 \\
55 \\
31 \\
14 \\
7 \\
15\end{array}$ & $\begin{array}{l}\text { Stillborn } \\
3.34 \text { days } \\
4.2 \text { wk } \\
10.7 \text { wk } \\
4.9 \text { mth } \\
7.6 \mathrm{mth} \\
10.1 \text { mth } \\
3 \mathrm{yr}\end{array}$ & $\begin{array}{l}(20 \mathrm{~min}-14 \mathrm{~d}) \\
(15 \mathrm{~d}-6 \mathrm{wk}) \\
(7-15 \mathrm{wk}) \\
(4-6 \mathrm{mth}) \\
(7-8 \mathrm{mth}) \\
(9-12 \mathrm{mth}) \\
(13 \mathrm{mth}-10 \mathrm{yr})\end{array}$ & $\begin{array}{l}0.14 \\
0.24 \\
0.04 \\
0.6 \\
0.45 \\
0.52 \\
2.3 \\
2.9\end{array}$ & $\begin{array}{l}(0-2) \\
(0-1) \\
(0-1) \\
(0-9) \\
(0-2) \\
(0-3) \\
(0-9) \\
(0-7)\end{array}$ & $\begin{array}{c}0.33 \\
0 \cdot 75 \\
1 \cdot 7 \\
5 \cdot 5 \\
6 \cdot 1 \\
10 \cdot 2 \\
13 \cdot 0 \\
24 \cdot 7\end{array}$ & $\begin{array}{l}(0-3) \\
(0-11) \\
(0-13) \\
(0-24) \\
(0-20) \\
(3-33) \\
(1-41) \\
(5-88)\end{array}$ & $\begin{array}{l}0 \\
2 \cdot 1 \\
4 \cdot 4 \\
41 \cdot 7 \\
58 \cdot 1 \\
64 \cdot 3 \\
57 \cdot 1 \\
86 \cdot 6\end{array}$ & $\begin{array}{l}0 \\
4 \cdot 2 \\
4 \cdot 4 \\
31 \cdot 8 \\
29 \cdot 0 \\
57 \cdot 1 \\
57 \cdot 1 \\
80 \cdot 0\end{array}$ & $\begin{array}{l}0 \\
0 \\
2 \cdot 1 \\
8 \cdot 7 \\
8 \cdot 3 \\
16 \cdot 1 \\
14 \cdot 3 \\
42 \cdot 9 \\
26 \cdot 6\end{array}$ & $\begin{array}{c}0 \cdot 09 \\
25 \cdot 5 \\
39 \cdot 1 \\
68 \cdot 3 \\
77 \cdot 4 \\
92 \cdot 8 \\
85 \cdot 7 \\
100\end{array}$ & $\begin{array}{c}0 \cdot 09 \\
14 \cdot 9 \\
26 \cdot 1 \\
48 \cdot 3 \\
41 \cdot 9 \\
57 \cdot 1 \\
57 \cdot 1 \\
93 \cdot 3\end{array}$ \\
\hline
\end{tabular}

Table The number of lungs studied and the prevalence of lymph nodes and lymphoreticular aggregates found in the standard section from the right middle lobe

L.R. AGGREGATES

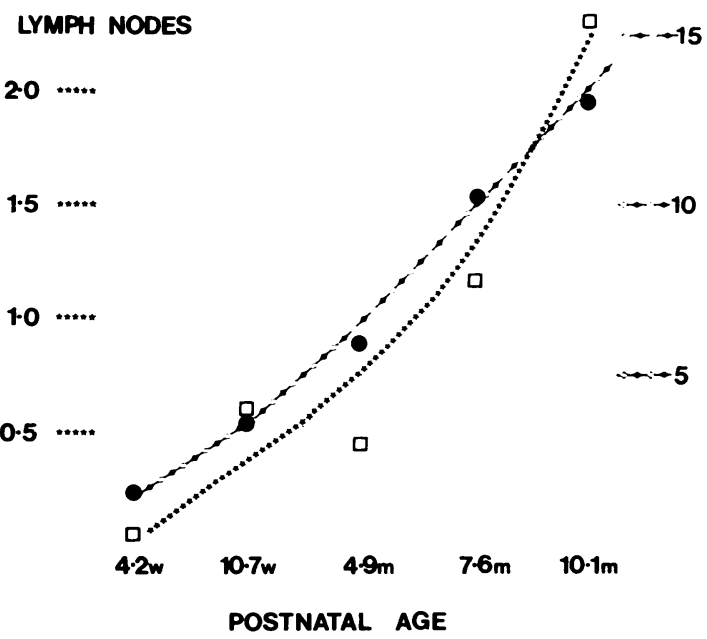

Fig 8 A graph showing the average number of lymph nodes and lymphoreticular aggregates in the standard right middle lobe section at different ages after birth. The starred line against clear squares with the scale on the left refers to nodes and that on the right with solid black dots to aggregates.

\section{LYMPHORETICULAR AGGREGATES}

In the overall series no peripheral aggregates were seen in the right middle lobe section from the 21 lungs from children stillborn or dying within 24 hours of birth. Two lungs only showed peripheral aggregates in the age group birth to 2 weeks, and these were in children aged 8 and 12 days, and only one lung from the 23 lungs examined from children between the ages of 3 and 6 weeks contained alveolar aggregates. Over that age the presence of these aggregates became increasingly plentiful but a small proportion of lungs appeared to be free from such aggregates at all ages.
When the rest of the lobes of the lung were surveyed it was extremely unusual for the right middle lobe section to have not recorded the presence of peripheral alveolar aggregates when such aggregates were present in other lobes. In almost all lungs if aggregates were missing in the sample section they were usually missing throughout the whole lung.

These peripheral aggregates usually contain a few capillaries and most showed some change in the local alveolar epithelium. This varied from simple plumping of the local cells to the presence of areas of almost columnar cells. Local alveoli also contain a few free alveolar epithelial cells.

When particles of carbon are present in these lungs they do not occur within the peripheral lymphoreticular aggregates. Aggregates and collections of carbon can be situated close together in the lung fields, but the carbon lying in cells in the connective tissue shows no associated collection of reactive cells.

It is necessary to separate the aggregates occurring in the connective tissue from those among alveoli as they are often partly surrounded by lymph channels and some of them may represent developing true lymph nodes. The general pattern of incidence of these non-alveolar aggregates is the same as that of the alveolar aggregates. However, they appear at a younger age group and were invariably present in the lungs of all children of 6 months of age or older.

The most common site for the presence of these aggregates is in the connective tissue between the final smallest air passage lined by ciliated and cuboid epithelium and its accompanying artery. These aggregates also appear at the junction where small bronchioles divide. Their general structure is slightly more compact than those seen in the alveolar walls and there is usually a fairly large lymph sinus coursing around one side of the aggregates.

We see here two groups of structures, one early developing lymph nodes and the other aggregates 


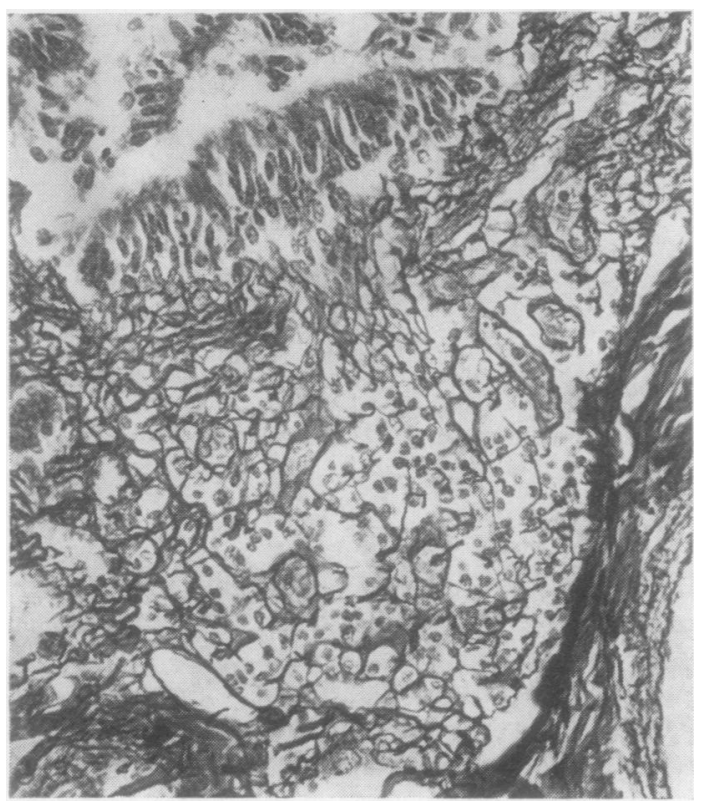

Fig 9 A lymphoreticular aggregate situated in the area between an artery and a bronchiole and stained for reticulin showing the relation of the aggregate to the epithelium of the bronchiole $(\times 315)$.

situated at the starting point of a definite lymph channel (fig 4).

Lymphoreticular aggregates in the bronchi occur characteristically within the mucosa and just deep to the epithelium. When an aggregate is seen deep in the wall of a bronchus, in particular when deep to a piece of cartilage, it is extremely common to find a termination of a mucous gland right in the centre of the aggregate. In some lungs the lymphoreticular aggregates formed virtually confluent rings within the bronchi and epithelium but these did not occur in any of the non-pathological lungs. When aggregates occur beneath the mucous membrane of the bronchi the overlying epithelial cells usually appear different from the epithelial cells in the rest of the perimeter of the bronchus, being less columnar and having a more vesicular nucleus (fig 9). In the abnormal lungs the cells at these sites occasionally show inclusion bodies.

\section{Discussion}

The purpose of this study was to determine whether or not lymphoreticular aggregates are present in the lungs of normal newborn children, their general pattern of development and probable significance, and to determine whether or not the presence of lymphoreticular aggregates within the lungs has the same significance in infants as in newborn piglets. The decision as to whether or not a lung was pathological was made without any knowledge of the lymphoreticular scoring of the right middle lobe and was made on the basis of the whole of the postmortem findings, including the sections of all lobes of the lungs. Many lungs containing much lymphatic and lymphoreticular tissue were removed from the series because of lung pathology as were some perinatal lungs where there was some obvious intrauterine infection and septicaemia. In this selection the presence or absence of lymphoreticular aggregates in the lung was deliberately not used as a criterion for exclusion or inclusion of a case. We thus have as far as possible a population of lungs in which any structure present could be taken as being incidental.

Formed and populated lymph nodes are present in all lungs at the time of birth but peripheral lymphoreticular aggregates are absent at birth in the normal lung and make their first appearance at about the end of the first week, and from this time onwards appear in increasing numbers. As the peripheral lymphoreticular aggregates appear to be of approximately the same size throughout the first year after birth, the increased number seen in the standard right midlobe sections indicates a progressive and steady increase in the number of these aggregates.

In assessing the number of nodes a direct comparison between scores on individual sections is only valid between lobes of different ages if the size of the structure scored remains constant. Thus a doubling of the individual size of the same number of lymph nodes may lead to at least a doubling in the number of nodes likely to be included in a single cross section. This subject is further complicated by the general enlargement of the whole structure of the lung. Since we have no data on these points we are not able accurately to assess the number of lymph nodes. Thus this apparent eightfold increase in the number of nodes could be false and does not imply an essential increase of this extent in the number of nodes.

The situation regarding the diagnosis of lymph nodes is further complicated by the difficulty in diagnosing unpopulated nodes and forming nodes in the perinatal period. There is thus the possibility that some forming nodes could have been either missed altogether or misdiagnosed as small lymphoreticular aggregates lying within the connective tissue. This means that the number of small lymph nodes and developing lymph nodes is likely to be underestimated in the very young lung. In lungs which showed no lymph nodes in the standard 
section of the right middle lobe, when the sections from the rest of the lung were surveyed in all cases, including stillborns, lymph nodes were found.

The sites of lymph nodes in the perinatal lung appeared to be different from those seen in older lungs, in that in the perinatal lungs the nodes seemed to be more prominent in the pleura and peripheral connective tissues than in the older lungs. This is probably due to many of the lymph nodes near the main bronchi in the very young children being small and relatively unpopulated. The impression gained is that in the perinatal lung the lymph nodes are almost uniformly distributed throughout the pleura, connective tissue, and hilum, and that it is only later after the development of lymphoreticular aggregates in the lung that the lymph nodes related to the bronchi and bronchioles become populated and obvious.

The number and postnatal development of lymph nodes was not the primary objective of this study. Our attention was drawn acutely to the problem of diagnosing the presence of unpopulated lymph nodes and the possibility of lymph nodes forming after birth in relation to lymphoreticular aggregates. The question of the possibility of induction of new lymph nodes after birth has yet to be elucidated.

The alveolar lymphoreticular aggregates were all small and they did not appear to increase in size in the older children, and thus the problem of size influencing the scoring of aggregates in single sections seems unlikely to be of great importance, and certainly not analagous to the lymph node situation. In none of the alveolar aggregates in this series did we see the development of Flemming centres.

Our findings in the human lung appear to coincide almost identically with the picture found by Jericho in his piglets that had been born by caesarean section into a germ-free environment. Jericho showed that the presence of lymphoreticular aggregates was directly the result of stimulation by biologically derived antigens and the appearance of infants' lungs would suggest that exactly the same situation applies. That carbon particles occur in the lungs of infants quite separate from lymphoreticular aggregates also lends support to this thesis.

In the work carried out by Jericho the number and extent of peripheral lymphoreticular aggregates bore a direct relationship to the microbiological concentration of antigen in the animals' environment.
We have long been able to differentiate fairly clearly what type of house or locality a child of 1 or 2 years came from, from the presence or absence of soot within the lung tissues. The presence of soot in the lung is an index of a child's immediate smoky environment. It would thus seem rational to extend this concept to the presence or absence of lymphoreticular aggregates and to use this as a measure of the concentration of biologically derived antigens. At the moment we are unable to identify the antigen. The possibility arises that in a number of infants this may be related to such factors as cows' milk as well as mites, bacteria, and viruses. The next step is to devise techniques to identify the antigens responsible for particular lymphoreticular aggregates.

Work on this study was partly supported by the Endowment Fund of the United Sheffield Hospitals, the Richard Fund, and the Foundation for the Study of Infant Deaths.

Photographs by Mr Alan Tunstill.

\section{References}

Bastianini, A. (1967). Osservazioni sulla mor fologia microscopica e l'isotopografia dei vase linlatici del polmone umano. Boll. Soc. ital. Biol. sper., 43, $1567-70$.

Bryant, B. J., and Shifrine, M. (1972). Histogenesis of lymph nodes during development of the dog. Journal of Reticuloendothelial Society, 12, 96-107.

Collet, A., and Policard, A. (1962). Essai de localisation infrastructurale dans le poumon des éléments du système réticuloendothélial. C.R. Soc. Biol. (Paris), 156, 991-995.

Emery, J. L., and Wilcock, P. F. (1966). The post-natal development of the lung. Acta Anat. (Basel), 65, 10-29.

Harvey, D. F., and Zimmerman, H. M. (1935). Studies on the development of the human lung. I. The pulmonary lymphatics. Anat. Rec., 61, 203-229.

Hayek, H. (1960). The Human Lung. Hofner, New York.

Jelinek, and Jelinkova, V. (1957). Reaction of the lymphatic tissue in lungs and their diagnostical importance. Sborn. Cesk. Akad., Zemed. vet. Med., 2, 379-394.

Jericho, K. W. F. (1970). Intrapulmonary lymphoid tissue of healthy pigs. Res. vet. Sci., 11, 548-552.

Jericho, K. W. F., Austwick, P. K. C., Hodges, R. T., and Dixon, J. B. (1971). Intrapulmonary lymphoid tissue of pigs exposed to aerosols of carbon particles, of Salmonella Cranienburg, of Mycoplasma granularum and to an oral inoculum of larvae of Metastrogylus apri. J. comp. Path., 81, 13-21.

Jericho, K. W. F., Derbyshire, J. B., and Jones, J. E. T. (1971). Intrapulmonary lymphoid tissue of pigs exposed to aerosols of haemolytic streptococcus group $\mathbf{L}$ and porcine adenovirus. J. comp. Path., 81, 1-11.

Lauweryns, J. M. (1971). Stereomicroscopic funnel-like architecture of pulmonary lymphatic valves. Lymphology, 4, 125-132.

Miller, W. S. (1911). The distribution of lymphoid tissue in the lung. Anat. Rec., 5, 99-119.

Tobin, C. E. (1957). Human pulmonic lymphatics. Anat. Rec., 127, 611-633.

Yoffey, J. M., and Courtice, F. C. (1970). Lymphatics, Lymph, and the Lymphomyeloid Complex, p. 47. Academic Press, London. 